\title{
Effect of p38 MAPK Inhibition on Apoptosis Marker Expression in the Process of Peritoneal Adhesion Formation
}

\author{
Irina A. Shurygina, $\mathrm{PhD}, \mathrm{ScD}^{1 *}$; Nataliya I. Aushinova, $\mathrm{PhD}^{1}$; Michael G. Shurygin, $\mathrm{PhD}, \mathrm{ScD}^{2}$ \\ ${ }^{1}$ Irkutsk Scientific Center of Surgery and Traumatology, ${ }^{2}$ Pharmasyntez \\ Irkutsk, the Russian Federation
}

\begin{abstract}
Background: Apoptosis, one of the most important mechanisms for maintaining homeostasis, is carried out under both physiological and pathological conditions. The aim of our study was to investigate the expression of markers of apoptosis through caspase-dependent and caspase-independent pathways during the reparative regeneration after serosal injuries of the peritoneum in the context of the prolonged p38 MAPK inhibition.

Methods: Peritoneal adhesions in the animal models were induced by a method developed by the authors that included opening the serous-muscular layer of the caecum with a $1 \mathrm{~cm}$ cut followed by closing the wound with a Schmieden suture and scarifying a $1.5 \times 1.5 \mathrm{~cm}$ area of the parietal peritoneum of the right lateral channel. Experiments were carried out on male 9-monthold Wistar rats. When closing the wound, control animals were intraperitoneally administered $3 \mathrm{~mL}$ of $0.9 \%$ sodium chloride solution $(n=40)$ while experimental group rats were administered slow-release drug Seroguard ${ }^{\circledR}$ (Pharmasyntez JSC) $(n=40)$. Animals were sacrificed within the period of 2 hours to 30 days post surgery. Expression of apoptosis markers was studied by immunohistochemical (Bcl-2, Bcl-xl) and immunofluorescent (PARP-1) staining.

Results: It is interesting that, in cases of the natural regeneration of the peritoneal injury, expression of anti-apoptosis markers at the injury site came in two waves: it was the most pronounced on days 1-3 post surgery while the second peak of activity was observed on day 14 . Within this time window, granulation tissue was actively growing and mature connective-tissue vascularized adhesions were being formed. By the end of the observation period (day 30), expression of anti-apoptosis proteins at the injury site became extremely low and a significant reduction in the amount of connective tissue cells was observed. It was found that a prolonged inhibition of the p38 activity resulted in a moderate increase in Bcl-2 expression on days 3-7, and a decrease in the activity on day 14 was followed by another increase in expression by day 30 . The Bcl-xl expression was observed 12 hours to 3 days post surgery and then it went down to the minimum. Positive PARP-1 staining observed on days 3 to 30 , which reached its maximum on day 14 , was also typical of the experimental group.

Conclusion: The performed study demonstrated that a prolonged p38 MAPK inhibition in the adhesion formation models results in the activation of fibroblast apoptosis at the reparation site, which, in the authors' opinion, predetermines a significant decrease in the adhesion formation in the experimental group.(International Journal of Biomedicine. 2018;8(4):342-346.)
\end{abstract}

Key Words: adhesions $\bullet$ peritoneal cavity $\bullet$ p38 MAPK $・$ apoptosis $・$ Seroguard $\bullet B c l-2 ・ B c l-x l ・ P A R P-1$

\section{Abbreviations}

AIF, apoptosis-inducing factor; Bcl-2, B-cell lymphoma 2; Bcl-xl, B-cell lymphoma-extra large; MAPK, mitogen-activated protein kinase; PARP-1, poly-[ADP-ribose]-polymerase-1.

\section{Introduction}

Apoptosis, one of the most important mechanisms for maintaining homeostasis, is carried out under both physiological and pathological conditions. Currently, the main mechanisms of apoptosis development in eukaryotic cells have been characterized and the research into apoptosis activators and regulators is underway. The interest in this issue lies in an opportunity to apply knowledge about the programmed cell death in medicine for the treatment of various diseases..$^{(1)}$

The Bcl family of proteins play an important role in regulating apoptosis, and among them there are both pro- 
(Bax, Bad, Bok, Bcl-xS, Bak, Bid, Bik, Bim, Krk, Mtd) and antiapoptotic types (Bcl-2, Bcl-xL, Bcl-w, Mcl-1, A-1, Boo). ${ }^{(2)}$ It is assumed that a ratio of anti-apoptotic and pro-apoptotic proteins is crucial to actualization of the programmed cell death. The Bcl-2 family of proteins regulate apoptosis mainly at the mitochondrial signaling pathway ${ }^{(3,4)}$ since signals of receptor-dependent signaling pathways generally bypass Bcl-2-mediated control. Bcl-2, Bcl-xL, and Bax proteins are capable of forming trans-membrane channels or being involved in their formation. For example, Bcl-2 directly or indirectly inhibits cytochrome $\mathrm{C}$ release from mitochondria ${ }^{(5)}$ while Bax complexed with porin, on the contrary, forms a channel in the outer mitochondrial membrane for the release of cytochrome $\mathrm{C}$ and AIF into the cytoplasm. Apart from their channel-forming activity, proteins of the Bcl-2 family can act as adaptors that bind to proteins involved in apoptosis.

Since the Bcl-2 family of proteins is the key regulator of apoptosis, the abnormalities in its function have been implicated in many diseases, including cancer, neurodegenerative disorders, and autoimmune diseases. ${ }^{(6)}$

Systems that regulate DNA integrity are also important for apoptosis. PARP-1(cleaved p85) is known to participate in the repair of DNA damage. After DNA is cleaved by enzymes participating in the cell death (such as caspases), PARP-1 can deplete the ATP of a cell in an attempt to repair the damaged DNA. ATP depletion in a cell leads to the death of that cell. Moreover, PARP-1 has an ability to induce apoptosis directly via the production of poly(ADP-ribose), which stimulates mitochondria to release AIF. This mechanism appears to be caspase-independent. ${ }^{(7,8)}$

Apoptosis also plays a crucial part in the reparative regeneration processes. In particular, control over cellularity of the tissue being formed at the wound healing site is mediated by apoptosis. ${ }^{(9,10)}$

In the present research into the role that mitogenactivated protein kinase cascades play in regulating reparative regeneration after a peritoneal injury, the study of the relationship between the anti- and pro-apoptotic signal activity and the MAPK activity is certainly of interest.

Objective: to investigate the expression of markers of apoptosis through caspase-dependent and caspaseindependent pathways during the reparative regeneration after serosal injuries of the peritoneum in the context of the prolonged p38 MAPK inhibition.

\section{Materials and Methods}

Peritoneal adhesion simulation

Peritoneal adhesions in the animal models were induced by a method developed by the authors that included opening the serous-muscular layer of the caecum with a $1 \mathrm{~cm}$ cut followed by closing the wound with a Schmieden suture and scarifying a $1.5 \times 1.5 \mathrm{~cm}$ area of the parietal peritoneum of the right lateral channel. ${ }^{(11-13)}$ Experiments were carried out on male 9-month-old Wistar rats ( $\mathrm{n}=80$ ) weighing $220-250 \mathrm{~g}$.

Animals were divided into 2 groups:

1) The control group $(\mathrm{n}=40)$ - intraperitoneal administration of $3 \mathrm{~mL}$ of $0.9 \%$ sodium chloride solution warmed to $37^{\circ} \mathrm{C}$ when closing the wound $(n=40)$;

2) The experimental group $(n=40)$ - intraperitoneal administration of $3 \mathrm{~mL}$ of the slow-release drug Seroguard ${ }^{\circledR}$ (Pharmasyntez JSC), i.e., a sterile aqueous solution of conjugate of 4-(4-Fluorophenyl)-2-(4-methylsulfinylphenyl)5-(4-pyridyl)-1H-imidazole ${ }^{(14)}$ with polyvinyl imidazole, warmed to $37^{\circ} \mathrm{C}$ when closing the wound $(\mathrm{n}=40)$. Seroguard ${ }^{\mathbb{}}$ 's main mechanism of action is a prolonged inhibition of the p38 MAPK activity in cells of the peritoneal surface layers.

The volume of fluid administered intraperitoneally was calculated in accordance with the data on the minimal volume needed to completely cover the peritoneum. ${ }^{(15)}$ Animals were sacrificed within the period of 2 hours to 30 days post surgery (after 2, 6, 12, and 24 hours; on days 3, 7, 14, and 30).

Animals were housed in keeping with the rules for good laboratory practice (GLP). The experiments were performed in accordance with the norms for the humane treatment of animals, which are regulated by the International Guidelines of the Association for the Assessment and Accreditation of Laboratory Animal Care in accordance with the protocol approved by the Institutional Animal Care and Use Committee of the Irkutsk Scientific Center of Surgery and Traumatology.

\section{Immunomorphological studies}

Tissue material (intestinal suture area, adhesion attachment sites, peritoneum) was fixed in FineFix solution (Milestone, Italy). After fixation, tissues were processed, embedded in paraffin blocks, and cut into $5 \mu \mathrm{m}$ serial sections.

Then sections were deparaffinized. To study apoptosis marker expression, preparations were IHC stained with Bcl-2 primary antibodies (Abbiotec, Cat. N 250555, Lot 09110202) in a dilution of 1:300, Bcl-xl primary antibodies (Epitomics, Cat. N 1018-3, Lot E-07-12-01) in a dilution of 1:100, and Novolink Polymer System secondary antibodies (Novocastra, $\mathrm{REF}=7112$, Lot 6006512) labeled with peroxidase. Sections were counterstained with $0.02 \%$ hematoxylin solution. Immunofluorescent staining ${ }^{(16)}$ was carried out with the use of PARP-1 primary antibodies (Epitomics, Cat. N 1074s, Lot CO11822) in a 1:50 dilution and Alexa Fluor 568 labeled secondary antibodies (Invitrogen, Cat. N A-11036 Lot 757102 ) in a 1:300 dilution. Tissue sections were visualized under a Nikon Eclipse 80i research microscope configured with DIH-M epi-fluorescence attachment.

\section{Results}

Expression of Bcl-2 family proteins during the reparative regeneration in animal models with serosal injury of the peritoneal cavity was studied both in the context of natural wound healing and under p38 MAPK inhibition by Seroguard ${ }^{\circledR}$ developed by the authors.

An increased expression of Bcl-2 and Bcl-xl was observed in control animals 2 hours after inducing adhesion formation. Single cells in the intestinal suture area and submucosal cells at the injury site were positively stained. No changes were observed 6 hours post surgery: Bcl-2 and Bcl$\mathrm{xl}$ were expressed by single cells at the peritoneal injury site.

The intensity of staining for Bcl-2 marker expression 
increased significantly by 12 hours post surgery while we also observed neutrophil infiltration around the suture, neutrophil infiltration and edema of the peritoneum, fibrin accumulation in the peritoneum and thickening of it, neutrophil and eosinophil infiltration of the submucosal intestinal layer, edema, and hemorrhages. Numerous cells at the peritoneal inflammation site were brightly stained for Bcl-2. Positive staining for Bcl$\mathrm{xl}$ was also observed: Some cells in the peritoneal injury site were weakly stained while distinct staining was observed in the cytoplasm of cells at the intestinal suture area.

One day after surgery, the intensity of Bcl-2 and Bcl-xl staining was still growing in control animals against the backdrop of adhesion formation, pronounced neutrophil infiltration of the suture area and the submucosal and muscular layer of the intestinal wall, and pronounced neutrophil infiltration of the peritoneum. Peritoneal cells at the adhesion formation site were brightly stained for both markers, with many positively stained cells observed in the submucosal layer.

Three days post surgery, in the control group there were many cells positively stained for Bcl-2 found in the submucosal layer and at the adhesion formation site (Figure 1).

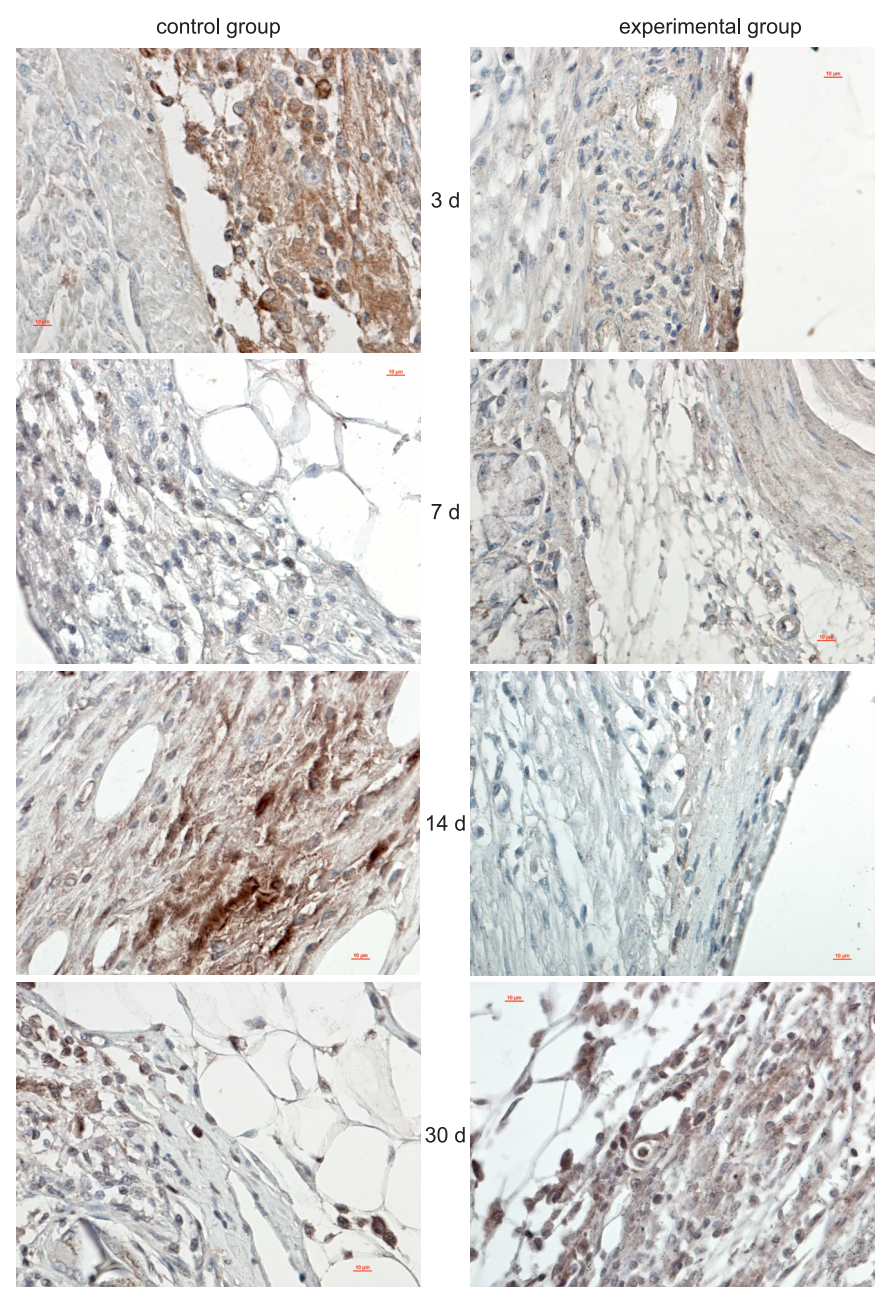

Fig.1.

Bcl-2 expression in control and experimental animals; immunohistochemistry with primary antibodies Bcl-2 (Abbiotec), 1:300, hematoxylin counterstaining.
At the same time, adhesions were being formed between the intestine and the omentum, between the site of the abdominal wall injury and the omentum, and between the abdominal wall and the intestine; and pronounced inflammation around the intestinal suture, widespread infiltration of the submucosal and muscular intestinal layer, evident signs of peritoneal inflammation, and growth of granulation tissue at the adhesion formation site (i.e., beginning of the fibroblastic phase of wound healing) were observed. Bcl-xl primary antibodies provided a very bright staining that was observed in the peritoneum inflammation area and the submucosal layer (Figure 2).

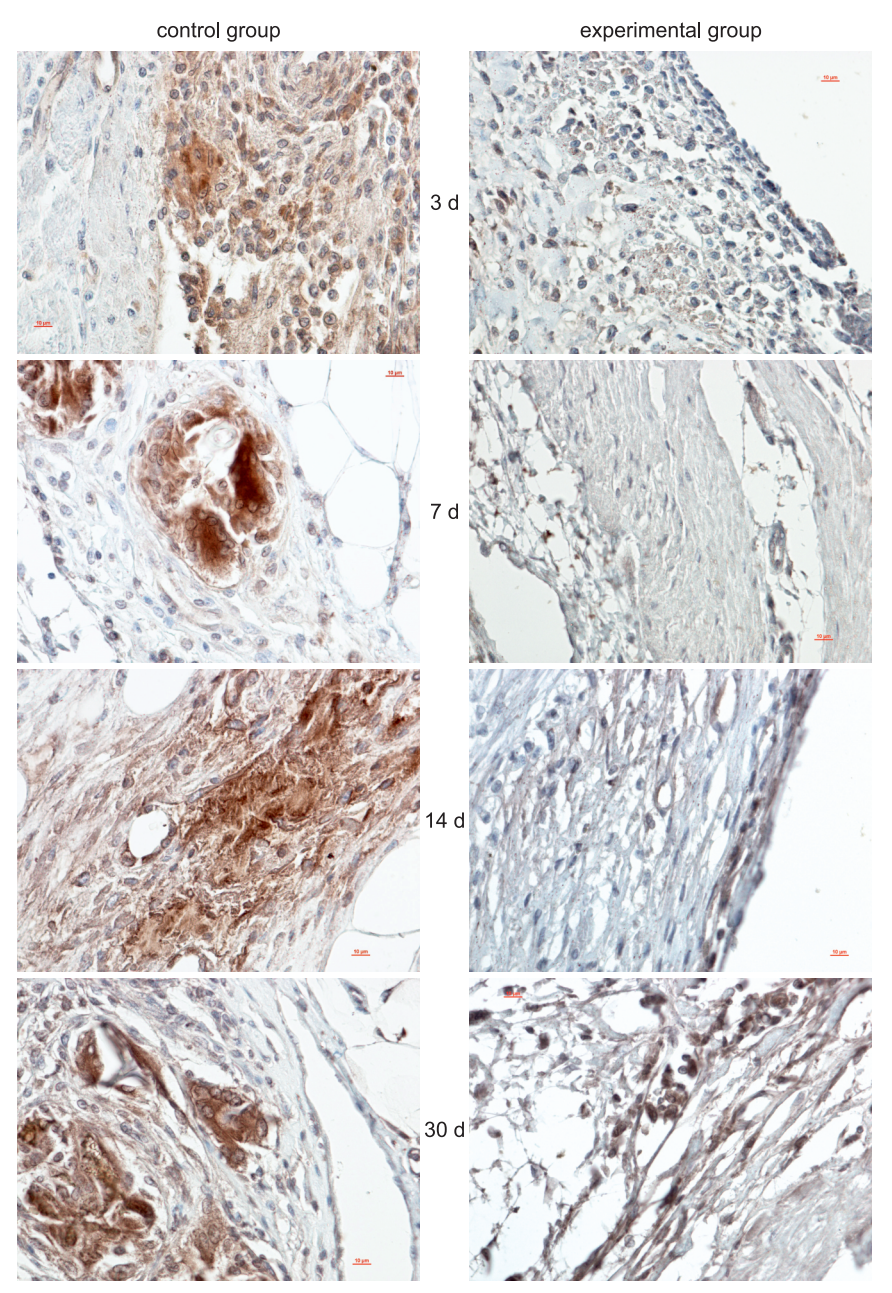

Fig.2.

Bcl-xl expression in control and experimental animals, immunohistochemistry with primary antibodies Bcl-xl (Epitomics), 1:100, hematoxylin counterstaining.

On day 7, rather bright Bcl-xl staining and weak Bcl2 staining were observed against the backdrop of dense and long adhesion formation with no pronounced vascularization or presence of the capsule around the suture. What's more, the zones of staining for the two markers were different. In particular, an area of inflammation around the intestinal suture was positively stained for Bcl-2 while positive staining for Bcl-xl was observed in another area of the intestinal suture 
inflammation that was different from the Bcl-2 positive one, and in the peritoneum, where it was bright.

On day 14, a very pronounced expression of Bcl-2 and Bcl-xl was still observed in submucosal cells, at the site of adhesion formation, and at the site of the anterior abdominal wall injury. Dense vascularized adhesions were being formed and connective tissue around the suture was growing. By day 30 , in control animals Bcl-2 staining at the site of proliferation was minimal, and the same area was brightly stained for Bcl-xl against the backdrop of formation of multiple highly vascularized adhesions with a high density of collagen fibers.

The Bcl-2 and Bcl-xl expression in the experimental group differed from that observed in the control group. In particular, no positive staining for Bcl-2 and Bcl-xl was observed 2 hours after inducing adhesion formation. After 6 and 12 hours post surgery, we observed intestinal wall hemorrhages at the injury site and peritoneum deserosation with a moderately expressed inflammation of the area. Only single cells at the peritoneal injury site were positively stained for Bcl-2, with positive staining also observed around the suture while Bcl-x primary antibodies provided brighter staining of these areas.

After 24 hours, in the experimental group a moderate Bcl-2 staining was observed in the peritoneum at the adhesion site as well as around the intestinal suture with a moderate expression of $\mathrm{Bcl}-\mathrm{xl}$ found in the same areas. Adhesions were formed between the intestine and the omentum, which was slightly attached to the suture site, and the peritoneum was moderately inflamed.

On day 3, the suture area and submucosal layer were brightly stained for $\mathrm{Bcl}-2$ while $\mathrm{Bcl}-2$ staining of the peritoneum was weak. All the three areas gave very weak Bcl-xl positive staining. There was minimal inflammation around the intestinal suture with a low cell packing density, mild inflammation at the peritoneal injury site, and the area of adherence between internal organs involved in the adhesion formation was minimal.

On day 7, a minimal Bcl-2 and Bcl-xl expression was observed. At the same time, short loose adhesions were being formed between either the intestinal suture and the omentum or the laparotomy wound and the omentum, and a mildly pronounced inflammation was observed.

On day 14, in the experimental group, no positive Bcl2 staining was observed against the backdrop of sporadic adhesions in the form of a slight attachment of the intestinal suture or the laparotomy wound to the omentum and minimal inflammation of the intestinal suture. Cells at the suture area as well as in the peritoneum were weakly stained for Bclxl. On day 30, moderate staining for Bcl-2 was observed in the experimental group against the backdrop of single filmy unvascularized adhesions between the intestinal suture and the omentum with minimal contact and poorly developed connective tissue at the adhesion site. The suture area was brightly stained for Bcl-x while staining at the adhesion site was moderate (Figures 1 and 2).

To study expression of PARP-1(cleaved p85), we employed immunofluorescent staining. It was found that expression of PARP-1 could be observed in control animals only on day 7 after surgery, or later, whereas in the earlier periods of time there was no specific staining. On day 7, multiple PARP-1 positive cells were found in the submusocal layer with some single cells found at the peritoneal injury site (Figure 3).
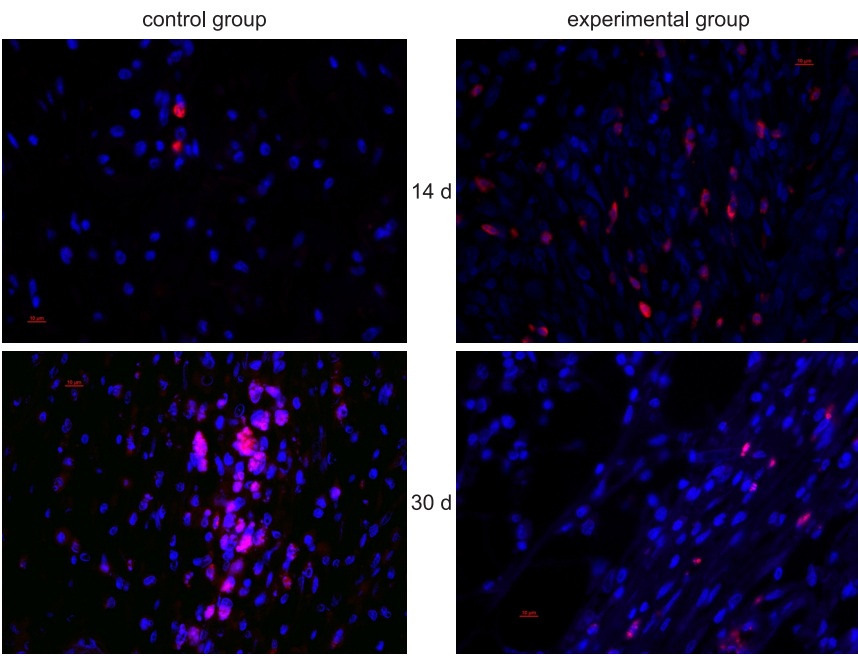

Fig.3.

PARP-1 expression; immunofluorescent staining; PARP-1 (cleaved p85) primary antibodies (Epitomics), 1:50, Alexa Fluor 568 labeled secondary antibodies, Dapi.

The number of positively stained cells at the site of adhesion formation increased, reaching its maximum by day 30 .

In the experimental group, single positively stained cells were observed at the peritoneal injury site on days 3 and 7 after inducing adhesion formation. On day 14, we observed numerous PARP-1 positive cells at the suture area and some sporadic cells in the submucosal layer (Figure 3). On day 30, there were still a fairly large number of positively stained cells at the adhesion formation site, around the intestinal suture, and in the submucosal layer. Thus, the activation of apoptosis in animals of the experimental group was identified.

\section{Discussion}

Surgical treatment methods are widely used in current medical practice, and a big share of the surgical procedures is made up of approaches that suppose an invasion comes in the form of a major surgery, thus making it impossible to avoid damaging the serous membrane lining the cavity. So taking the above facts into consideration, we have performed some experimental studies aimed at clarifying pathogenesis of the serous lining reaction to an injury and evaluating a potential effect of p38 MAPK inhibition on the mechanisms of apoptosis.

Apoptosis is known to be one of the most important mechanisms for maintaining homeostasis. Moreover, its mechanisms are carried out under both physiological and pathological conditions.

The studies of the apoptotic activity during adhesion formation provide controversial data. Binnebösel et al. ${ }^{(17)}$ carried out a prospective study of 40 patients with adhesive disease after abdominal surgery and found apoptosis activation in all cases. 
At the same time, G.M. Saed et al. ${ }^{(18)}$ studied cell lines derived from 5 patients with adhesive disease using polymerase chain reaction and found that fibroblasts from adhesions manifested sharply decreased levels of apoptosis marker expression compared to normal fibroblasts. In the authors' opinion, a decrease in the apoptosis activity could be indirectly responsible for the intensity of adhesion formation.

We have studied the expression of Bcl-2 family proteins during the reparative regeneration in animal models with serosal injury of the peritoneal cavity both in the context of natural regeneration process and under p38 MAPK inhibition.

We have found that in cases of a traumatic lesion of the peritoneum, pro-apoptotic and anti-apoptotic processes run simultaneously. While anti-apototic mechanisms prevail (more intense Bcl-2 staining) in the initial post-injury period, caspasedependent pro-apoptotic phenomena take the lead at the later stages (more intense Bcl-xl staining). The PARP-1 activation indicates an increase in the frequency of cell DNA damage while the duration of that process induces the cell death via a caspaseindependent pathway. Taken together, these processes result in eliminating a large number of cells, primarily fibroblastic ones, from the area of the aseptic inflammation at the peritoneal injury site where connective tissue is being formed.

It is interesting that, in cases of the natural regeneration of the peritoneal injury, expression of anti-apoptosis markers at the injury site came in two waves: it was the most pronounced on days 1-3 post surgery while the second peak of activity was observed on day 14 . Within this time window, granulation tissue was actively growing and mature connective-tissue vascularized adhesions were being formed. By the end of the observation period (day 30), expression of anti-apoptosis proteins at the injury site became extremely low and a significant reduction in the amount of connective tissue cells was observed. ${ }^{(19)}$

It was found that a prolonged inhibition of the p38 activity resulted in a moderate increase in Bcl-2 expression on days 3-7, and a decrease in the activity on day 14 was followed by another increase in expression by day 30 . The Bcl-xl expression was observed 12 hours to 3 days post surgery and then it went down to the minimum. Positive PARP-1 staining observed on days 3 to 30 , which reached its maximum on day 14 , was also typical of the experimental group.

The performed study demonstrated that a prolonged p38 MAPK inhibition in the adhesion formation models results in the activation of fibroblast apoptosis at the reparation site, which, in the authors' opinion, predetermines a significant decrease in the adhesion formation in the experimental group.

\section{Conflict of interest}

The authors declare that they have no competing interests.

\section{References}

1. Karp G. Cell and molecular biology: Concepts and experiments. 6th ed. John Wiley\&Sons, Hoboken, NY; 2010.

*Corresponding author: Prof. Irina A. Shurygina, PhD, ScD. Irkutsk Scientific Center of Surgery and Traumatology. Irkutsk, the Russian Federation, E-mail: irinashurygina@gmail.com
2. Gordeeva AV, Labas YA, Zvyagilskaya RA. Apoptosis in unicellular organisms: mechanisms and evolution. Biochemistry (Mosc). 2004;69(10):1055-66.

3. Popgeorgiev N, Jabbour L, Gillet G. Subcellular Localization and Dynamics of the Bcl-2 Family of Proteins. Front Cell Dev Biol. 2018; 6:13. doi: 10.3389/fcell.2018.00013. 4. Kale J, Osterlund K, Andrews DW. BCL-2 family proteins: changing partners in the dance towards death. Cell Death Differ. 2018;25(1):65-80. doi: 10.1038/cdd.2017.186.

5. Chao DT, Korsmeyer SJ. BCL-2 family: regulators of cell death. Annu Rev Immunol. 1998;16:395-419.

6. Siddiqui WA, Ahad A, Ahsan H. The mystery of BCL2 family: Bcl-2 proteins and apoptosis: an update. Arch Toxicol. 2015;89(3):289-317. doi: 10.1007/s00204-014-1448-7.

7. Yu SW, Andrabi SA, Wang H, Kim NS, Poirier GG, Dawson TM, Dawson VL. Apoptosis-inducing factor mediates poly(ADP-ribose) (PAR) polymer-induced cell death. Proc Natl Acad Sci U S A. 2006;103(48):18314-9.

8. Ling XX, Liu JX, Zhou L, DU YJ, Chen SQ, Chen JL et al. Poly(ADP-ribosyl)ation of Apoptosis Antagonizing Transcription Factor Involved in Hydroquinone-Induced DNA Damage Response. Biomed Environ Sci. 2016;29(1):80-4. doi: 10.3967/bes2016.008.

9. Desmoulière A, Redard M, Darby I, Gabbiani G. Apoptosis mediates the decrease in cellularity during the transition between granulation tissue and scar. Am J Pathol. 1995; 146(1):56-66.

10. Hinz B. Formation and function of the myofibroblast during tissue repair. J Invest Dermatol. 2007;127(3):526-37.

11. Ayushinova NI, Lepekhova SA, Shurygina IA, Roj TA, Shurygin MG, Zaritskaja LV, Gol'dberg OA. Method for simulating abdominal commissures. Patent RU 2467401, 27.07.2011.

12. Ayushinova NI, Shurygina IA, Shurygin MG, Balykina AV, Malgataeva ER, Popova AD, Yankelevich SA. Experimental model for developing of ways to prevent adhesions in the abdominal cavity. Siberian Med J. 2012;109(2):51-53.

13. Shurygina IA, Shurygin MG, Rodionova LV, Aushinova NI. Interleukin expression in the area damaged by the development of abdominal cavity adhesions. International Journal of Biomedicine. 2017;7(4):293-297. doi: 10.21103/Article7(4)_OA4.

14. Shurygin MG, Shurygina IA. Compounds, pharmaceutical compositions and a method for the prophylaxis and treatment of the adhesion process. Patent WO2012156938, 20.03.2014. 15. diZerega GS. Peritoneum, peritoneal healing and adhesion formation. In: diZerega GS, editor. Peritoneal surgery. BerlinHeidelberg-New York: Springer; 2006:3-38.

16. Shurygin MG, Shurygina IA, Dremina NN, Kanya $\mathrm{OV}$. Endogenous progenitors as the source of cell material for ischemic damage repair in experimental myocardial infarction under conditions of changed concentration of vascular endothelial growth factor. Bull Exp Biol Med. 2015; 158(4):528-31. doi: 10.1007/s10517-015-2801-4.

17. Binnebösel M, Klinge U, Rosch R, Junge K, Lynen-Jansen P, Schumpelick V. Morphology, quality, and composition in mature human peritoneal adhesions. Langenbecks Arch Surg. 2008;393(1):59-66.

18. Saed GM, Jiang Z, Fletcher NM, Diamond MP. Modulation of the BCL-2/BAX ratio by interferon-gamma and hypoxia in human peritoneal and adhesion fibroblasts. Fertil Steril. 2008; 90(5):1925-30.

19. Shurygina IA, Shurygin MG, Ayushinova NI. Expression of apoptosis markers in adhesions in the abdominal cavity under the experimental conditions. Vestn Ross Akad Med Nauk. 2014;(5-6):29-33. 